\title{
Dinámica del sistema afianzador de México: una aplicación de diagramas de fase
}

\section{Dynamics of the mixing system of Mexico: An application of phase diagrams}

\author{
Nora Gavira-Durón ${ }^{1}$, Ana Lorena Jiménez Preciado ${ }^{2}$, Einar Moreno Quezada ${ }^{1}$ \\ ${ }^{1}$ Universidad de las Américas Puebla, México \\ ${ }^{2}$ Instituto Politécnico Nacional, México
}

Recibido el 24 de julio de 2017; aceptado el 19 de abril de 2018

Disponible en Internet el: 4 de diciembre de 2018

\section{Resumen}

A partir de la implementación de Solvencia II en México, se generó un cambio significativo en la normatividad para aseguradoras y afianzadoras, lo que implicó incremento en sus costos y gastos, en particular para el sector afianzador, ya que les representa una mayor carga regulatoria con respecto a su Ley anterior; teniendo un impacto directo en el costo de las primas y la generación de utilidades. El objetivo del presente trabajo es analizar la dinámica entre el total de primas emitidas por el sector afianzador y la utilidad bruta reportada por las mismas; lo anterior permite determinar si la captación de primas es suficiente para incrementar las utilidades reportadas por el sector. Se utilizan diagramas de fase con la finalidad de comprender la dinámica temporal entre las variables y conocer sus trayectorias en relación al Estado Estacionario. A partir de la metodología propuesta se señala que las afianzadoras mexicanas, a pesar de sus utilidades, presentan comportamientos globalmente inestables, asimismo, se verifican los casos donde una mayor aversión al riesgo de las afianzadoras (representadas por las primas emitidas) no siempre reflejan mayores utilidades.

Código JEL: C02, C39, C62, G22

Palabras clave: Solvencia II; Afianzadoras mexicanas; Diagramas de fase

\footnotetext{
*Autor para correspondencia

Correo electrónico: aseunam53@yahoo.com.mx (N. Gavira Durón)

La revisión por pares es responsabilidad de la Universidad Nacional Autónoma de México. 


\begin{abstract}
Since the application of Solvency II in Mexico, it has been implanted significant changes in regulations for insurers and sureties, which implied an increase in costs and expenses, particularly for the surety sector, since it represents a more significant regulatory burden in regard to the previous Law. This new regulation has a direct impact on insurance premium and its profits. In that sense, the aim of this paper is to analyze the dynamics between total insurance premiums and net income reported of the surety sector in Mexico; this allow to determine whether the collection of insurance premiums is enough to increase net incomes. Phase diagrams are used to recognize the temporal dynamics between variables and know their trajectories in relation to the Steady State. Based on the proposed methodology, it is noted that surety companies present globally unstable behaviors. Likewise, it is verified cases where increased risk aversion of the sureties (represented by the premiums) do not always reflect higher earnings.
\end{abstract}

JEL Code: $\mathrm{C} 02, \mathrm{C} 39, \mathrm{C} 62, \mathrm{G} 22$

Keywords: Solvency II; Mexican surety; Phase diagrams

\title{
Introducción
}

Los inicios de Solvencia surgen con la Primera Directiva de No Vida (PDC, 1973), en donde se establecen los requisitos de margen de solvencia y en 1979 con la Primera Directiva de Vida (PDV, 1979), mismas que tuvieron diversas modificaciones, hasta que en 1992 se crea la regulación única para el mercado de seguros, llamada Tratado de Solvencia, con el objetivo de establecer normas para que las instituciones de seguros cuenten con un adecuado margen de solvencia y establecer los requisitos mínimos de capital. Con lo que, al momento de que se promulgó del Tratado de la Unión Europea ${ }^{1}$, ya se contaba con uno de los mercados de seguros más competitivos a nivel mundial.

En la búsqueda de homologar los principios de Basilea III, con lo establecido en Solvencia y después de varios años de trabajo, el 30 de septiembre de 2015, la Comisión Europea encargada de Solvencia, formuló una serie de enmiendas, con lo que se generó Solvencia II (Commission, 2017), que engloba el proyecto de revisión del régimen de suficiencia de capital para la industria aseguradora europea. Solvencia II es una serie de normas que abarcan, entre otras cosas: valoración de activos y pasivos, niveles de capital de acuerdo a los riesgos asumidos, gestión de riesgos y gobernanza; evaluación de equivalencia entre regímenes de solvencia de terceros países y el marco modelo interno.

Para el caso de México, en enero de 1935 entra en vigor la Ley Sobre el Contrato de Seguro (Cámara de Diputados d. H., 1935.) y en diciembre de 1950, la Ley Federal de Ins-

\footnotetext{
${ }^{1}$ El cual forma parte de los tratados bajo los que posteriormente se regiría la Unión Europea.
} 
tituciones de Fianzas (Cámara de Diputados d. H., 1950), mismas que por décadas siguieron vigentes y después de un significativo número de reformas, el 04 de abril de 2013 se publica en el Diario Oficial de la Federación la Ley de Instituciones de Seguros y Fianzas (LISF), (Cámara de Diputados d. H., 2013), basada en Solvencia II, misma que entró en vigor en México, de forma paulatina, a partir del 04 de abril de 2015 para todo el sector asegurador y afianzador. En dicho lineamiento se establece que la Comisión Nacional de Seguros y Fianzas (CNSF) es la encargada de emitir las disposiciones a que se refiere dicha Ley, con anterioridad al inicio de su vigencia, por lo que el 09 de diciembre de 2014 se publica en el Diario oficial de la Federación, la Circular Única de Seguros y Fianzas (CUSF) (Comisión Nacional de Seguros y Fianzas, 2014). La implementación en México, representa un cambio significativo con respecto a la regulación anterior, en particular para las afianzadoras, ya que esta figura no existe en Solvencia II.

Siendo un problema la nueva regulación para las afianzadoras, ya que les representa una mayor carga regulatoria, se propone un análisis para el sector afianzador desde el enfoque de control óptimo, en específico, utilizando diagramas de fase. Dicha herramienta se ha utilizado generalmente en sistemas químicos y en robótica como se muestra en los trabajos realizados por (Belmonte, Morales, Fernández-Caballero, \& Somolinos, 2016) dónde presentan el diseño de un control multivariado no lineal descentralizado para un helicóptero de laboratorio no lineal y multivariado; (Zoujun, 2016) diseña modelos para un sistema de control en red con retraso de tiempo incierto. (Saini, Kumar, \& Rajput, 2016) presentan una metodología de control en cascada para el reactor químico exotérmico no lineal, (Hongbin, Jian, \& Yuelieng, 2016) aplican un algoritmo de control de aprendizaje iterativo (ILC) de tipo abierto al sistema discreto no lineal de un robot móvil. (Chessab, 2016) sugiere un controlador difuso para usar en la determinación y control de Kufasat equipado con tres bobinas magnéticas. Mientras que (Álvarez \& Londoño, 2014) proponen un método de control de velocidad angular para mejorar la acción de un controlador en un proceso de transformación de papel. En lo que respecta a los temas económicos, son ampliamente utilizados en los modelos de expectativas racionales, en específico, en temas monetarios donde se trata de explicar la dinámica de los precios a partir de las decisiones de política monetaria, dichos trabajos se pueden encontrar en (Muth, 1961), (Lucas \& Sargent, 1981), (Sargent \& Wallace, 1973), (Chow, 1983) así como también en la propuesta del modelo IS-LM ampliado y dinámico planteado por (Blanchard, 1981); así como (Laitner, 1990) utiliza los diagramas de fase para para modelar cambios de impuestos, por dar unos ejemplos. Sin embargo, no se han presentado investigaciones relacionadas meramente con el sector financiero, en particular, del sector afianzador.

Entre los estudios que abordan el análisis del sector afianzador a partir de los cambios propuestos en Solvencia II, los podemos encontrar en (Sirmans \& Mccullough, 2017) en donde se señala la necesidad de aumentar la información de los usuarios que adquieren servicios 
de aseguradoras y afianzadoras con la finalidad de mejorar los ratings o consideraciones que se toman en cuenta para dos cuestiones: 1) para proporcionar el servicio de seguro/fianza y segundo, para determinar la cantidad o porcentaje que se debe entregar a partir de la información que proporcionan los clientes. Asimismo, (Eling \& Pankoke, 2014) estudian la regulación de los mínimos requerimientos de capital del sector asegurado en general de la Unión Europea a través de la implementación de Solvencia II, entre sus hallazgos más destacables -implementando la metodología de Valor en Riesgo (VaR)- se comprueban desviaciones sustanciales que al final terminan provocando reducciones en los requisitos de capital para las empresas europeas seleccionadas.

En el caso de (Zimmer, Grundl, Schade, \& Glenzer, 2016), se enfocaron en analizar la reacción de una muestra de 281 personas aseguradas ante el pago de contrato que presentaba diferentes niveles de riesgo. En sus conclusiones destacan que los individuos, más que presentar aversión al riesgo, toman sus decisiones de contratación tomando decisiones ambiguas, mismo que se puede ver reflejado en un aumento de en la probabilidad de incumplimiento y en un cambio en la demanda de seguros.

En otro estudio de (Braun, Schmeiser, \& Schreiber, 2017), evalúan los rendimientos esperados y los efectos de diversificación de una muestra de diferentes empresas europeas a partir los requerimientos de Solvencia II, los resultados sugieren que la medición planteada por el estándar de Solvencia II puede repercutir en el cumplimiento de las obligaciones contractuales de las aseguradoras, provocando que sus inversiones se inclinen en activos riesgosos con la finalidad de que puedan dar cumplimiento a la mínima reserva de capital que se les solicita. Sin embargo, para el caso de México, los estudios del sector afianzador ${ }^{2}$ se reducen a un puñado de investigaciones mismas que podemos encontrar, en su mayoría, en los boletines y reportes de la CNSF así como los análisis de las agencias calificadoras de riesgo. Por ejemplo, en el reporte de FitchRatings elaborado por (Martínez \& Turrubiartes, 2017), indica que, a partir de los cambios implementados en Solvencia II, la utilidad técnica de las aseguradoras y afianzadoras se ha visto beneficiada, sin embargo, una de las causas es que los mismos accionistas han inyectado recursos para que pueda aumentar el capital contable de las mismas.

En ese sentido, el objetivo de la presente investigación es analizar la dinámica entre el total de primas emitidas por el sector afianzador y la utilidad bruta reportada por las mismas para determinar si la captación de primas es suficiente para incrementar las utilidades reportadas por el sector de fianzas en México. Se utilizan diagramas de fase con la finalidad de comprender la dinámica temporal entre las variables, así como conocer sus trayectorias en relación al estado estable o estado estacionario. Se considera la utilidad bruta ya que se

\footnotetext{
${ }^{2} \mathrm{~A}$ diferencia del sector asegurador en donde si bien se contemplan más investigaciones, no es abundante la literatura que se especialice en el tema.
} 
quiere analizar el impacto económico que tendrá en el sector afianzador el incremento de reservas técnicas por los requerimientos de la nueva regulación en el sector afianzador y, de acuerdo a la Circular única de seguros y fianzas (CUSF) (CNSF, 2018), en su Anexo 22.1.2 "Criterios de Contabilidad aplicables a las instituciones, sociedades mutualistas y sociedades controladoras”, en la Serie III, “Criterios relativos a los estados financieros básicos consolidados", inciso "a" Catálogo Mínimo (C-1), en dónde se enlistan los conceptos que forman parte del Estado de Resultados de las Instituciones y Sociedades Mutualistas, en la sección 2 “Estado de Resultados”, se indica que:

Utilidad (Pérdida) Técnica = Primas emitidas - Primas cedidas - Primas de retención - Incremento neto de la reserva de riesgos en curso y de fianzas en vigor - Primas de retención devengadas - Costo neto de adquisición - Costo neto de siniestralidad, Reclamaciones y otras obligaciones pendientes de cumplir - Reclamaciones recuperadas del reaseguro y reafianzamiento no proporcional

Utilidad (Pérdida) Bruta = Utilidad (pérdida) técnica - Incremento neto de otras reservas técnicas - Resultado de Operaciones Análogas y Conexas

Además, la CUSF (CNSF, 2018), en su Título 21, Capítulo 21.1 indica que las operaciones análogas y conexas pertenecen únicamente a las instituciones de seguros; entonces, para el caso de las Afianzadoras.

Utilidad (Pérdida) Bruta = Utilidad (Pérdida) Técnica - Incremento neto de otras reservas técnicas La investigación está dividida en las siguientes secciones: se comienza por describir las características del sector afianzador en México así como la forma en como Solvencia II las afecta; en el siguiente apartado se abordan los aspectos metodológicos en donde se propone un sistema dinámico que captura la dinámica entre las primas emitidas y la utilidad bruta de cada afianzadora, misma que finalmente se reflejan en los diagramas de fase; el análisis de resultados se presenta en la subsecuente sección para finalmente dar pie a las conclusiones del documento.

\section{Integración del sector afianzador en México}

De acuerdo a la LISF, una institución de fianzas es la sociedad anónima autorizada para organizarse y operar como tal, siendo su objeto el otorgamiento de fianzas a título oneroso ${ }^{3}$. Se denomina fianza a un contrato por el cual una institución de fianzas (fiador) se compromete a garantizar el cumplimiento de obligaciones con contenido económico, contraídas por una

\footnotetext{
${ }^{3}$ De acuerdo a la definición de la Ley de Instituciones de Seguros y de Fianzas, artículo 2, apartado XVII.
} 
persona física o moral (cliente) ante otra persona física o moral privada o pública, en caso de que aquella no cumpliere. De acuerdo a cifras publicadas en el Boletín de Análisis Sectorial Seguros y Fianzas al 31 de diciembre de 2015 (Fianzas, 2015), el sector afianzador en México se conforma por 15 compañías, los ramos en que operan se muestran en la Tabla 1:

Tabla 1

Instituciones del Sector Afianzador, nombre y ramo

\begin{tabular}{|c|c|c|c|c|}
\hline COMPAÑÍA & $\begin{array}{c}\text { FIDELI- } \\
\text { DAD }\end{array}$ & $\begin{array}{l}\text { JUDI- } \\
\text { CIAL }\end{array}$ & $\begin{array}{l}\text { ADMINIS- } \\
\text { TRATIVAS }\end{array}$ & $\begin{array}{l}\text { CRÉ- } \\
\text { DITO }\end{array}$ \\
\hline ACE Fianzas Monterrey, S.A. & Sí & Sí & Sí & Sí \\
\hline Afianzadora Aserta, S.A. de C.V., Grupo Financiero Aserta & Sí & Sí & Sí & Sí \\
\hline Afianzadora Fiducia, S.A. de C.V. & No & No & Sí & No \\
\hline Afianzadora Insurgentes, S.A de C.V., Grupo Financiero & Sí & Sí & Sí & Sí \\
\hline \multicolumn{5}{|l|}{ Aserta } \\
\hline Afianzadora Sofimex, S.A. & Sí & Sí & Sí & Sí \\
\hline CESCE Fianzas México. S.A. de C.V. & No & Sí & Sí & No \\
\hline Chubb de México, Compañía Afianzadora, S.A. de C.V. & Sí & Sí & Sí & Sí \\
\hline Crédito Afianzador, S.A., Compañía Mexicana de Garantías & Sí & Sí & Sí & Sí \\
\hline Fianzas Asecam, S.A. & Sí & Sí & Sí & Sí \\
\hline Fianzas Atlas, S.A & Sí & Sí & Sí & Sí \\
\hline Fianzas Dorama, S.A. & Sí & Sí & Sí & Sí \\
\hline Fianzas Guardiana Inbursa, S.A., Grupo Financiero Inbursa & Sí & Sí & Sí & Sí \\
\hline Mapfre Fianzas S.A. & Sí & Sí & Sí & Sí \\
\hline Primero Fianzas, S.A. de C.V. & Sí & Sí & Sí & Sí \\
\hline Zurich Fianzas México & No & Sí & Sí & Sí \\
\hline
\end{tabular}

Fuente: Elaboración propia, datos de la Comisión Nacional de Seguros y Fianzas (CNSF).

La nueva Ley tiene por objetivo conservar la solvencia y estabilidad de las instituciones, fomentar una conducta de mercado adecuada considerando a los usuarios; así como el perfeccionamiento en los cálculos de valuación de las reservas y de las inversiones, a una mayor difusión de la información respectiva a los consejeros, accionistas, al mercado y a las autoridades supervisoras. Es decir, la nueva regulación implica la imposición de un costo relativamente alto asociado a los requerimientos de un gobierno corporativo y su funcionamiento (Gavira \& Castillo, 2017). Bajo este esquema, las afianzadoras pequeñas, que por lo regular son de capital puramente nacional y no pertenecen a grupos financieros, son las que mayor impacto tienen al operar conforme a lo establecido en la nueva regulación, lo que a su vez presenta un 
impacto directo en consumidores y por consiguiente en el costo de las primas; mientras que instituciones con matrices extranjeras cuentan con ciertas ventajas al implementar la nueva normatividad, con lo que podrán sacar mayor ventaja a las pequeñas instituciones.

$\mathrm{Al}$ cierre de 2015, de las 15 instituciones de fianzas registradas ante la CNSF, 6 son instituciones filiales que no pertenecen a un grupo financiero, 3 instituciones cuentan con capital nacional que pertenecen a un grupo financiero y 6 son instituciones de capital nacional que no pertenecen a un grupo financiero. Las 10 instituciones que tienen capital nacional y las 6 de ellas que no pertenecen a grupos financieros, se muestran en la Tabla 2:

Tabla 2

Instituciones con capital mayoritariamente nacional, Sector Afianzador, al cierre de 2015

\begin{tabular}{l|l}
\hline COMPAÑíA & 2015 \\
\hline Afianzadora Aserta, S.A. de C.V., Grupo Financiero Aserta & CNGF \\
Afianzadora Punto Aserta, S.A. & CNGF \\
Afianzadora Fiducia, S.A. de C.V. & $\mathrm{CN}$ \\
Afianzadora Insurgentes, S.A de C.V., Grupo Financiero Aserta & $\mathrm{CNGF}$ \\
Afianzadora Sofimex, S.A. & $\mathrm{CN}$ \\
Crédito Afianzador, S.A., Compañía Mexicana de Garantías & $\mathrm{CN}$ \\
Fianzas Asecam, S.A. & $\mathrm{CN}$ \\
Fianzas Atlas, S.A & $\mathrm{CN}$ \\
Fianzas Dorama, S.A. & $\mathrm{CN}$ \\
Fianzas Guardiana Inbursa, S.A., Grupo Financiero Inbursa & $\mathrm{CNGF}$ \\
\hline
\end{tabular}

$\mathrm{CN}$ : Instituciones con capital mayoritariamente nacional.

CNGF: Instituciones con capital mayoritariamente nacional y forman parte de Grupo Financiero.

Fuente: Elaboración propia, datos de la Comisión Nacional de Seguros y Fianzas (CNSF).

Con respecto al sector asegurador mexicano, al cierre de 2015, el total de las primas emitidas fue de 395,082.7 millones de pesos (98.3\% seguro directo), las primas directas de presentaron un incremento real anual de $7.3 \%$, con respecto al cierre del año anterior, por encima del crecimiento del PIB de $2.5 \%$. La utilidad neta del ejercicio, del sector asegurador fue de 21,214.4 millones de pesos $17.4 \%$ menor, en términos reales, con respecto al cierre del año anterior. Al cierre de 2015, las primas emitidas totales por el sector, ascendió a 9,494.1 millones de pesos (96.2\%).

Las primas directas del sector tuvieron un incremento real anual de $1.1 \%$ al cierre de 2015 , con respecto al mismo periodo anterior, este resultado se encuentra por debajo del 
crecimiento del PIB nacional que fue de $2.5 \%$, en el mismo periodo. La utilidad neta del sector en el ejercicio fue de 1,467.2 millones de pesos, lo que representa un decremento, en términos reales, de $11.6 \%$ respecto a lo reportado a fines de diciembre de 2014 . Considerando las primas emitidas en 2015, el sector de fianzas representa el $2.4 \%$ del sector asegurador y que la carga legar, técnica, de sistemas, reportes regulatorios, constitución de reservas, capital, etc., que representa la nueva regulación mexicana es la misma para ambos sectores; de ahí que represente un mayor impacto económico a las afianzadoras la implementación de dicha ley.

\section{Aspectos metodológicos}

La propuesta metodológica del presente trabajo consiste en utilizar diagramas de fase con la finalidad de comprender la dinámica temporal entre el total de primas emitidas por el sector afianzador y la utilidad bruta reportada por las mismas, asimismo, se exponen las trayectorias en relación al estado estable o estacionario. En la tabla 3 se presentan las afianzadoras mexicanas utilizadas en la presente investigación:

Tabla 3

Afianzadoras consideradas para el estudio

\begin{tabular}{l|l}
\hline Afianzadora & $\begin{array}{l}\text { Nombre } \\
\text { corto }\end{array}$ \\
\hline ACE Fianzas Monterrey, S.A. & $\begin{array}{l}\text { Monterrey } \\
\text { Aserta } \\
\text { Afianzadora Aserta, S.A. de C.V., Grupo Financiero Aserta } \\
\text { Afianzadora Fiducia, S.A. de C.V. }\end{array}$ \\
$\begin{array}{l}\text { Afianzadora Insurgentes, S.A. de C.V., Grupo Financiero } \\
\text { Aserta }\end{array}$ & Insurgentes \\
Afianzadora Sofimex, S.A. & Sofimex \\
Chubb de México, Compañía Afianzadora, S.A. de C.V. & Chubb \\
Crédito Afianzador, S.A., Compañía Mexicana de Garantías & Mexicana \\
Fianzas Asecam, S.A. & Asecam \\
Fianzas Atlas, S.A. & Atlas \\
Fianzas Dorama, S.A. & Dorama \\
Fianzas Guardiana Inbursa, S.A., Grupo Financiero Inbursa & Inbursa \\
Mapfre Fianzas, S.A. & Mapfre \\
Primero Fianzas, S.A. de C.V. & Primero \\
\hline
\end{tabular}

Fuente: Elaboración propia, datos de la Comisión Nacional de Seguros y Fianzas (CNSF). 
Los datos utilizados en el estudio proceden de la Comisión Nacional de Seguros y Fianzas (CNSF) a partir del segundo trimestre de 2007 al cuarto trimestre de 2015, con un total de 36 observaciones trimestrales por cada afianzadora, dando en total 468 observaciones disponibles (32 unidades por 11 momentos en el tiempo). Las variables utilizadas se muestran en la tabla 4:

Tabla 4

Variables propuestas para el estudio

\begin{tabular}{lcc}
\hline \multicolumn{1}{c}{ Variable } & Descripción & Unidades \\
\hline Prima & Primas emitidas & Miles de pesos \\
Utilidad & Utilidad bruta & Miles de pesos \\
\hline
\end{tabular}

Fuente: Elaboración propia, datos de la Comisión Nacional de Seguros y Fianzas (CNSF).

Las variables expresadas en la tabla 4 se deflactan a precios del último trimestre registrado del 2015, posteriormente, se trasforman las variables a índices de base fija siendo el periodo base también el último trimestre de la temporalidad del estudio; la finalidad de transformar los montos monetarios en índices es para facilitar la comparación y manejo de las cantidades.

El análisis de la dinámica del sistema de las afianzadoras comprendido por las variables prima y utilidad, parte de un sistema de ecuaciones diferenciales que requieren ser solucionadas de manera simultánea. Sea $\boldsymbol{x}=$ prima y $\boldsymbol{z}=\boldsymbol{u t i l i d a d}$ un sistema de dos ecuaciones diferenciales ordinarias de primer orden que toman la forma:

$$
\begin{aligned}
& \frac{d x}{d t}=\dot{x}=f(x, z, t) \\
& \frac{d z}{d t}=\dot{z}=g(x, z, t)
\end{aligned}
$$

Las funciones de solución son funciones diferenciables del tiempo $(t)$ para el sistema de ecuaciones diferenciales representados por (1) y (2). A continuación, se proponen las condiciones iniciales al sistema, que toman la forma:

$$
x_{0}=x\left(t_{0}\right) \quad y \quad z_{0}=z\left(t_{0}\right)
$$

Adicionalmente, se parte de una condición de equilibrio: sea $\left(\boldsymbol{x}^{*}, \mathbf{z}^{*}\right)$ un punto en el plano donde simultáneamente $\boldsymbol{f}\left(\boldsymbol{x}^{*}, \mathbf{z}^{*}\right)=\mathbf{0}$ y $\boldsymbol{g}\left(\boldsymbol{x}^{*}, \mathbf{z}^{*}\right)$, entonces se cumple que $\frac{d x}{d t}=\mathbf{0}$ y $\frac{d \boldsymbol{z}}{d t}=\mathbf{0}$ 
Lo anterior indica que las variables consideradas no cambian en el tiempo, en ese sentido, el sistema tiene un punto fijo o un punto de equilibrio, también conocido como estado estacionario o estado estable.

Retomando el sistema planteado en (1) y (2), se pueden obtener las ecuaciones diferenciales para las variables, es decir tanto para el prima $(\boldsymbol{x})$ como para la utilidad $(\boldsymbol{z})$. En ese sentido, el sistema dinámico se expresa de la forma siguiente:

$$
\begin{aligned}
& \dot{x}=\theta x+\beta z+j \\
& \dot{z}=\gamma x+\delta z+k
\end{aligned}
$$

Donde las ecuaciones en (4) y (5) describen el movimiento de las variables endógenas a lo largo del tiempo mientras que $\boldsymbol{\theta}, \boldsymbol{\beta}, \boldsymbol{\gamma}$ y $\boldsymbol{\delta}$ son los coeficientes asociados a las variables endógenas $\boldsymbol{x}, \boldsymbol{z}$. En ambas ecuaciones y representan las constantes del sistema. En la ecuación (4), las variaciones de $\dot{\boldsymbol{x}}$ o de las primas de las afianzadoras dependen positivamente de ella misma y positivamente de la variable endógena utilidad, análogo a $\dot{z}$ que también depende positivamente de las variables endógenas ${ }^{4}$.

Para cumplir con la condición de equilibrio donde $\frac{d x}{d t}=\mathbf{0}$ y $\frac{d z}{d t}=\mathbf{0}$, se igualan a cero las ecuaciones (4) y (5) y se despeja la segunda variable endogena en terminos de $\boldsymbol{x}$ para que las ecuaciones puedan solucionadas de manera simultánea, de esta manera se tiene:

$$
\begin{aligned}
& z=\frac{\theta}{\beta} x+\frac{j}{\delta} \\
& z=\frac{\gamma}{\delta} x+\frac{k}{\delta}
\end{aligned}
$$

Toda la información contenida en las ecuaciones diferenciales se une en un único gráfico, es este caso, en un diagrama de fases. Dichos diagramas tienen la particularidad de que se puede observar si un sistema se mueve hacia un punto fijo o equilibrio, o bien si, se aleja de él. En concreto, una trayectoria se aproxima a un punto fijo si $\boldsymbol{x}(\boldsymbol{t}) \rightarrow \boldsymbol{x}^{*}$ cuando, $\boldsymbol{t} \rightarrow \infty$ si es así, entonces se tiene un "atractor". Cuando $\boldsymbol{x}(\boldsymbol{t})$ se aleja de $\boldsymbol{x}^{*}$ cuando $\boldsymbol{t} \rightarrow \infty$, entonces se dice que es un "repulsor".

Sin embargo, también se puede dar la situación en donde la trayectoria se desplaza primero hacia el punto fijo y luego se aleja del estado estable, a esto se le conoce como "punto

\footnotetext{
${ }^{4}$ Las variaciones de $\boldsymbol{x}$ y $\boldsymbol{z}$ no siempre dependen positivamente de ellas mismas, esta situación la define el signo asociado a los coeficientes de las variables endógenas, dicho punto se aborda posteriormente en el apartado de estabilidad del sistema.
} 
de silla" o "corredor de estabilidad". En la Figura 1 se ilustran los tres diferentes casos que pueden presentar los diagramas de fase.
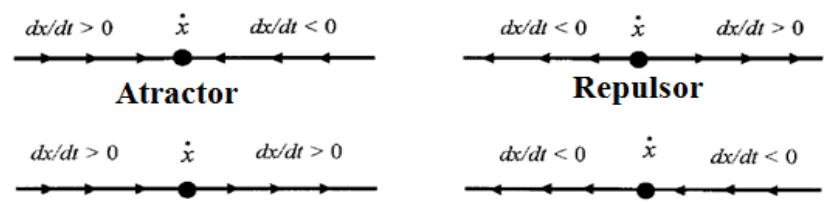

\section{Corredor de estabilidad o punto de silla}

Figura 1. Puntos fijos, atractores y repulsores.

Fuente: Adaptación de (Shone, 2002).

De esta manera, las flechas presentadas en la Figura 1 en los diagramas de fase muestran las regiones de las trayectorias se comportan como atractores, repulsores o puntos de silla respecto de , es decir, la dirección del cambio de cuando incrementa. Lo anterior constituye una parte fundamental del estudio pues permite conocer si el sistema dinámico es globalmente estable (cuando cualquier punto en el diagrama se comporta como un atractor), globalmente inestable (si cualquier punto del diagrama se comporta como un repulsor) o bien, si existe un corredor de estabilidad (cuando las direcciones son diferentes con respecto de ). Una vez explicado el sistema de ecuaciones diferenciales propuesto para el estudio, en el siguiente subapartado se analiza la estabilidad del sistema dinámico para cada afianzadora.

\section{Estabilidad del sistema}

Para conocer si los sistemas dinámicos propuestos para las afianzadoras presentan trayectorias estables o inestables, se considera conveniente especificar las ecuaciones diferenciales en términos de matrices y vectores, de esta manera se tiene:

$$
\left[\begin{array}{c}
\dot{\boldsymbol{x}} \\
\dot{\boldsymbol{z}}
\end{array}\right]=\boldsymbol{A}\left[\begin{array}{l}
\boldsymbol{x} \\
\boldsymbol{z}
\end{array}\right]+\left[\begin{array}{l}
\boldsymbol{j} \\
\boldsymbol{k}
\end{array}\right]
$$

Donde la matriz que contiene los coeficientes y se refieren a las constantes del sistema, en tanto que es la matriz de coeficientes asociados a las variables endógenas . Dichos coeficientes son los mismos expresados en las ecuaciones (4) y (5).

$$
\boldsymbol{A}=\left[\begin{array}{ll}
\boldsymbol{\theta} & \boldsymbol{\beta} \\
\boldsymbol{\gamma} & \boldsymbol{\delta}
\end{array}\right]
$$


Tomando en cuenta las condiciones iniciales, el punto de equilibrio se alcanza cuando $\frac{\boldsymbol{d} \boldsymbol{x}}{\boldsymbol{d} \boldsymbol{t}}=\mathbf{0}$ y $\frac{\boldsymbol{d z}}{\boldsymbol{d} \boldsymbol{t}}=\mathbf{0}$ esto es:

$$
\left[\begin{array}{l}
\dot{x} \\
\dot{y}
\end{array}\right]=\left[\begin{array}{l}
\mathbf{0} \\
\mathbf{0}
\end{array}\right]
$$

Para examinar la estabilidad del sistema se procede a calcular los valores propios asociados al sistema matricial planteado en la ecuación (8). Para sistemas homogéneos lineales, si el determinante de $\boldsymbol{A} \neq \boldsymbol{0}$, entonces el único punto de equilibrio se obtiene cuando $\boldsymbol{x}^{*}=\mathbf{0}$ y $\mathbf{z}^{*}=\mathbf{0}$ es decir:

$$
\begin{aligned}
\operatorname{Det}[\boldsymbol{A}-\lambda I] & =0 \\
\operatorname{Det} & =\left[\left[\begin{array}{ll}
\boldsymbol{\theta} & \boldsymbol{\beta} \\
\gamma & \delta
\end{array}\right]-\left[\begin{array}{ll}
\lambda & 0 \\
0 & \lambda
\end{array}\right]\right]=\operatorname{Det}\left[\begin{array}{cc}
\boldsymbol{\theta}-\lambda & \boldsymbol{\beta} \\
\gamma & \delta-\lambda
\end{array}\right]
\end{aligned}
$$

De lo anterior se obtiene una ecuación de la forma siendo la solución:

$$
\lambda_{1}, \lambda_{2}=\frac{-(\theta+\delta) \pm \sqrt{(\theta+\delta)^{2}-4(\theta \delta+\beta \gamma)}}{2}
$$

El signo asociado a las raíces depende de dos aspectos: en primera instancia, del signo previo a la raíz cuadrada y del signo que se observa una vez resuelta la raíz. Se resalta que la clave de los valores propios está el signo de la raíz, de manera concreta, los casos que se pueden obtener a partir del resultado de los signos asociados a los eigenvalores son los siguientes:

$$
\begin{array}{cc}
{\left[\begin{array}{ll}
- & \boldsymbol{\beta} \\
\boldsymbol{\gamma} & -
\end{array}\right]} & \text { Sistema globalmente estable } \\
{\left[\begin{array}{ll}
+ & \boldsymbol{\beta} \\
\boldsymbol{\gamma} & +
\end{array}\right]} & \text { Sistema globalmente inestable } \\
{\left[\begin{array}{ll}
+ & \boldsymbol{\beta} \\
\boldsymbol{\gamma} & -
\end{array}\right] \text { o }\left[\begin{array}{ll}
- & \boldsymbol{\beta} \\
\boldsymbol{\gamma} & +
\end{array}\right]} & \text { Punto de silla o corredor de estabilidad }
\end{array}
$$


Un sistema globalmente estable está asociado a signos negativos en la diagonal principal, en este caso, cualquier punto del diagrama de fase presenta un atractor. Asimismo, cuando los valores propios tienen asociados valores positivos entonces cualquier punto del diagrama de fase se aleja del estado estable, por lo que se tienen repulsores. Finalmente, el caso (15) hace alusión al corredor de estabilidad, donde las direcciones son diferentes con respecto de $\boldsymbol{x}^{*}$.

Cabe destacar que el concepto de estabilidad no insiste en que la trayectoria que siguen las variables tenga que acercarse al punto fijo de equilibrio. Como se ha mencionado con anterioridad, un punto fijo o de equilibrio $\left(\boldsymbol{x}^{*}, z^{*}\right)$ que satisface la condición $\boldsymbol{f}(\boldsymbol{x}, \boldsymbol{z})=\boldsymbol{0}$ y $\boldsymbol{g}(\boldsymbol{x}, \boldsymbol{z})=\boldsymbol{0}$ es estable o atractor dado algún valor $\left(\boldsymbol{x}_{\mathbf{0}}, \mathbf{z}_{\mathbf{0}}\right)$ cercano al punto $\left(\boldsymbol{x}^{*}, \boldsymbol{z}^{*}\right)$. De esta forma, los signos asociados a los valores propios determinan la estabilidad o inestabilidad del sistema. En el siguiente apartado se presentan los resultados obtenidos para cada afianzadora.

\section{Análisis de resultados: diagramas de fase}

Tras haber desarrollado el sistema de ecuaciones diferenciales propuesto para cada una de afianzadoras, se muestran los diagramas de fase que le corresponden a cada una y que cumplen con las condiciones de equilibrio planteadas. Se destaca que la matriz de coeficientes $\boldsymbol{A}$ planteada en la ecuación (9) no siempre presenta signos positivos en todos sus componentes, dicho aspecto es relevante pues define tanto la inclinación de las pendientes como el intercepto de las rectas, además del signo asociado a los valores propios.

Puesto que el sistema dinámico planteado se conforma por ecuaciones lineales, para los pares de datos asociados a las variables $(\boldsymbol{x}, \boldsymbol{z})$ de cada afianzadora, se calcula la desviación estándar de $\boldsymbol{x}$ y $\boldsymbol{z}$ y se representan con los símbolos $\boldsymbol{\sigma}_{\boldsymbol{x}}$ y $\boldsymbol{\sigma}_{\boldsymbol{y}}$ respectivamente. Posteriormente, se calcula el coeficiente de correlación lineal $\boldsymbol{p}$, que mide la fuerza y la dirección de una relación lineal entre prima y utilidad, de tal forma que cada coeficiente asociado a la matriz $\boldsymbol{A}$ representada por la ecuación (9) sigue en general la fórmula de la pendiente:

$$
\boldsymbol{\theta}, \boldsymbol{\beta}, \boldsymbol{\gamma}, \boldsymbol{\delta}=\boldsymbol{\rho} \frac{\sigma_{Z}}{\sigma_{x}}
$$

En la tabla (2) se muestran los coeficientes obtenidos ${ }^{5}$ en la matriz para cada una de las afianzadoras, es decir, se presentan los coeficientes asociados a . Si se observan con detenimiento los signos asociados a los coeficientes y, se puede conocer a priori si el sistema dinámico de cada entidad es estable o inestable, ya que y son los términos asociados para obtener los valores propios planteados en la ecuación (12).

5 Todos los cálculos que se presentan en el estudio, se realizan con el software R Project versión 3.3.0. Disponible en https:// www.r-project.org/. 
Tabla 5

Coeficientes asociados a la matriz A

\begin{tabular}{lcccccc}
\hline Afianzadoras & $\boldsymbol{\theta}$ & $\boldsymbol{\beta}$ & $\boldsymbol{\gamma}$ & $\boldsymbol{\delta}$ & $\boldsymbol{j}$ & $\boldsymbol{k}$ \\
\hline Monterrey & 0.6475 & 0.4851 & 0.2875 & 0.8890 & -0.6792 & -0.7286 \\
Aserta & 0.3177 & 0.6478 & -0.1024 & 0.8050 & -0.2745 & -0.1624 \\
Fiducia & 0.2604 & 0.1203 & -0.0790 & 0.9654 & -0.0030 & -0.0174 \\
Insurgentes & 1.1248 & -0.2282 & 0.4342 & 0.2439 & -0.2975 & -0.1691 \\
Sofimex & 0.0011 & 1.2762 & -0.3858 & 1.5047 & -0.5190 & -0.4658 \\
Chubb & 0.5692 & 0.0904 & -0.0174 & 0.7136 & -0.0721 & -0.0516 \\
Mexicana & 0.2318 & 0.2951 & -0.3217 & 1.1296 & -0.0109 & -0.0218 \\
Asecam & -0.1002 & 0.7983 & -0.6691 & 1.4433 & -0.0170 & -0.0177 \\
Atlas & 0.8772 & 0.2795 & 0.3686 & 0.7893 & -0.1172 & -0.1183 \\
Dorama & 0.8719 & 0.0708 & 0.4690 & 0.4784 & -0.1193 & -0.1218 \\
Inbursa & 0.8418 & 0.2484 & 0.0967 & 0.4235 & -0.4085 & -0.0834 \\
Mapfre & 0.4822 & 0.0882 & 0.0026 & 0.4686 & -0.0136 & -0.0047 \\
Primero & 0.9698 & 0.1589 & 0.4515 & 0.3864 & -0.1714 & -0.1139 \\
\hline
\end{tabular}

Fuente: Elaboración propia con salida de R-Project.

Dados los resultados de los coeficientes presentados en la Tabla 5 y observando los signos de los valores propios de las ecuaciones diferenciales que comprenden el sistema dinámico de cada afianzadora, se obtiene que solo la afianzadora Asecam presenta un corredor de estabilidad en tanto que las demás afianzadoras presentan un comportamiento inestable en cuanto a su relación prima-utilidad.

A continuación, se presentan los diagramas de fase que corresponde al comportamiento del sistema dinámico de cada afianzadora. Las flechas de los diagramas refieren a la dirección del cambio de $\mathbf{y}$ cuando. En la Figura 2 se presenta a la afianzadora Monterrey, la línea roja y la línea azul satisfacen el comportamiento de las ecuaciones (8) y (9) en donde y , es decir, parten de la condición de equilibwrio; el diagrama presenta un comportamiento globalmente inestable, ya que todas las flechas del diagrama son repulsores.

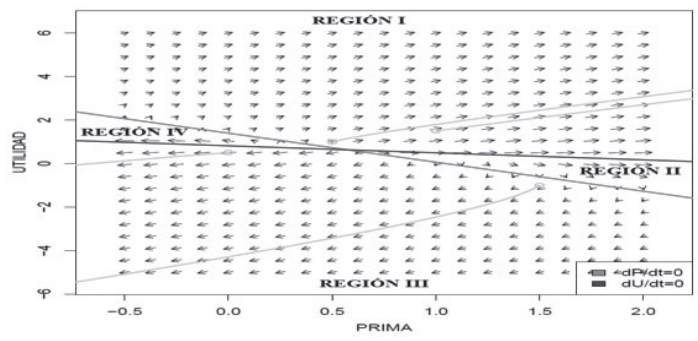

Figura 2. Diagrama de fase para Monterrey.

Fuente: Elaboración propia con base en

(Grayling, 2014) R-Project. 
Cabe señalar que los puntos con las curvas amarillas presentan coordenadas aleatorias que muestran los atractores/repulsores. Como se mencionó anteriormente, Monterrey presenta repulsores en todos sus puntos por lo que siempre se aleja del equilibrio, sin embargo, esto no necesariamente representa algo negativo. Por ejemplo, los valores correspondientes de prima en la región I, provocarían aumentos en la utilidad, pero, si los montos de las primas se movieran en la región II, tendríamos que mayores primas provocarían una disminución en la utilidad. En la región III y IV se observa que una estrategia de disminución de primas, lastimaría significativamente la utilidad de Monterrey. En cuanto a las trayectorias de las curvas, se sugiere que cuando la afianzadora toma una posición más agresiva o es más adversa al riesgo (aumentando el monto de sus primas) no se ve reflejada en una mayor utilidad, al contrario, provoca que comience a disminuir.

Adicionalmente, los diagramas de fases muestran la estrategia que utilizan las afianzadoras a partir de las trayectorias que siguen las ecuaciones diferenciales, por ejemplo, en el caso de Monterrey la pendiente de la curva de utilidades es negativa y toma tanto valores positivos como negativos; la pendiente de la curva de primas también es ligeramente negativa y lo que se observa es que en medida que la prima tiene un mayor valor las utilidades también aumentan. Bajo la misma lógica, en la Tabla 3 se presentan cuatro casos a analizar a partir de las pendientes de las trayectorias de las ecuaciones diferenciales:

Tabla 6

Casos a partir de las pendientes de las ecuaciones diferenciales $\dot{\boldsymbol{x}}_{\mathbf{1}}$ y $\dot{\boldsymbol{x}}_{\mathbf{2}}$

\begin{tabular}{cccc}
\hline Caso 1 & Pendiente & Caso 3 & Pendiente \\
\hline Utilidad & Positiva & Utilidad & Positiva \\
Prima & Positiva & Prima & Negativa \\
\hline Caso 2 & Pendiente & Caso 4 & Pendiente \\
\hline Utilidad & Negativa & Utilidad & Negativa \\
Prima & Positiva & Prima & Negativa \\
\hline
\end{tabular}

Fuente: Elaboración propia.

En el primer caso se tiene a afianzadora Asecam, la cual es la única que tiene pendientes positivas en sus trayectorias, en este caso se observa que la utilidad es elástica ante la variación de las primas, cuando Asecam es más adversa al riesgo, sus utilidades aumentan.

Lo anterior se puede apreciar en la Figura 3: 


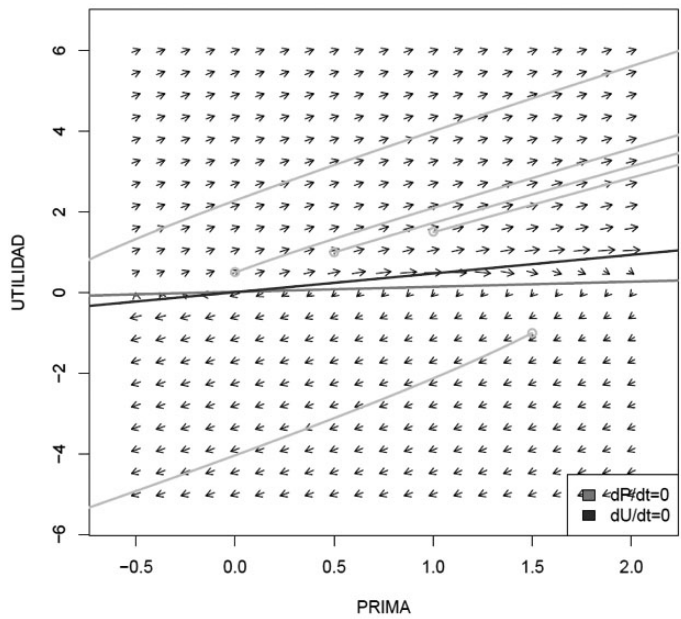

Figura 3. Diagrama de fase para Asecam.

Fuente: Elaboración propia con base en (Grayling, 2014) R-Project.

Para el siguiente caso, en donde la pendiente de la utilidad es negativa y el de la prima es positiva, solo la afianzadora Insurgentes presenta dicho comportamiento, lo que se observa es que cuando Insurgentes asume una postura más adversa al riesgo (elevando sus primas), sus utilidades tienden a caer de forma sensible, aunque, cuando las primas llegan al extremo de la región I, estas pueden llevar al sistema a un mayor nivel de utilidades. En la Figura 4 se presentan dicho comportamiento.

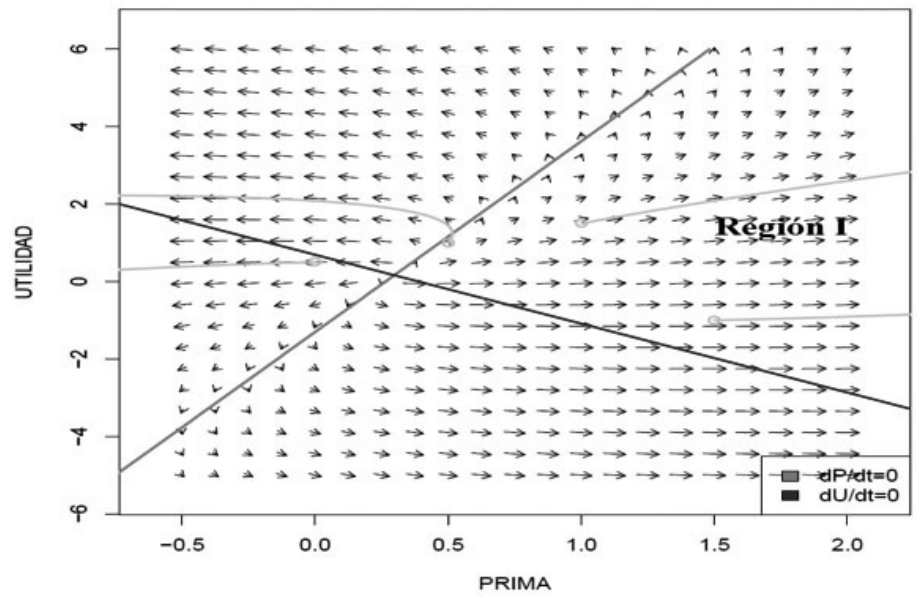

Figura 4. Diagrama de fase para Insurgentes.

Fuente: Elaboración propia con base en (Grayling, 2014) R-Project. 
Siguiendo las estrategias que pueden presentar las afianzadoras de acuerdo a la comparación de pendientes planteadas en la Tabla 6, cuando la pendiente de la utilidad es positiva y el de la prima es negativa, se tiene que la mayoría de las afianzadoras presentan dicho comportamiento: Aserta, Fiducia, Sofimex, Chubb Mexicana y Mapfre. En concreto, cuando las pendientes de las curvas de las afianzadoras mencionadas son más adversas al riesgo, se puede ver traducido en un mayor nivel de utilidad. Sin embargo, las afianzadoras Chubb, Fiducia y Mapfre, a pesar de que tienen las mismas pendientes del caso tres, difieren significativamente en su comportamiento ya que la trayectoria que sigue la utilidad está cerca de ser perfectamente elástica, por lo que estas afianzadoras son más sensibles ante variaciones de sus primas. A continuación, en la Figura 5, se presentan los diagramas de fase correspondientes.
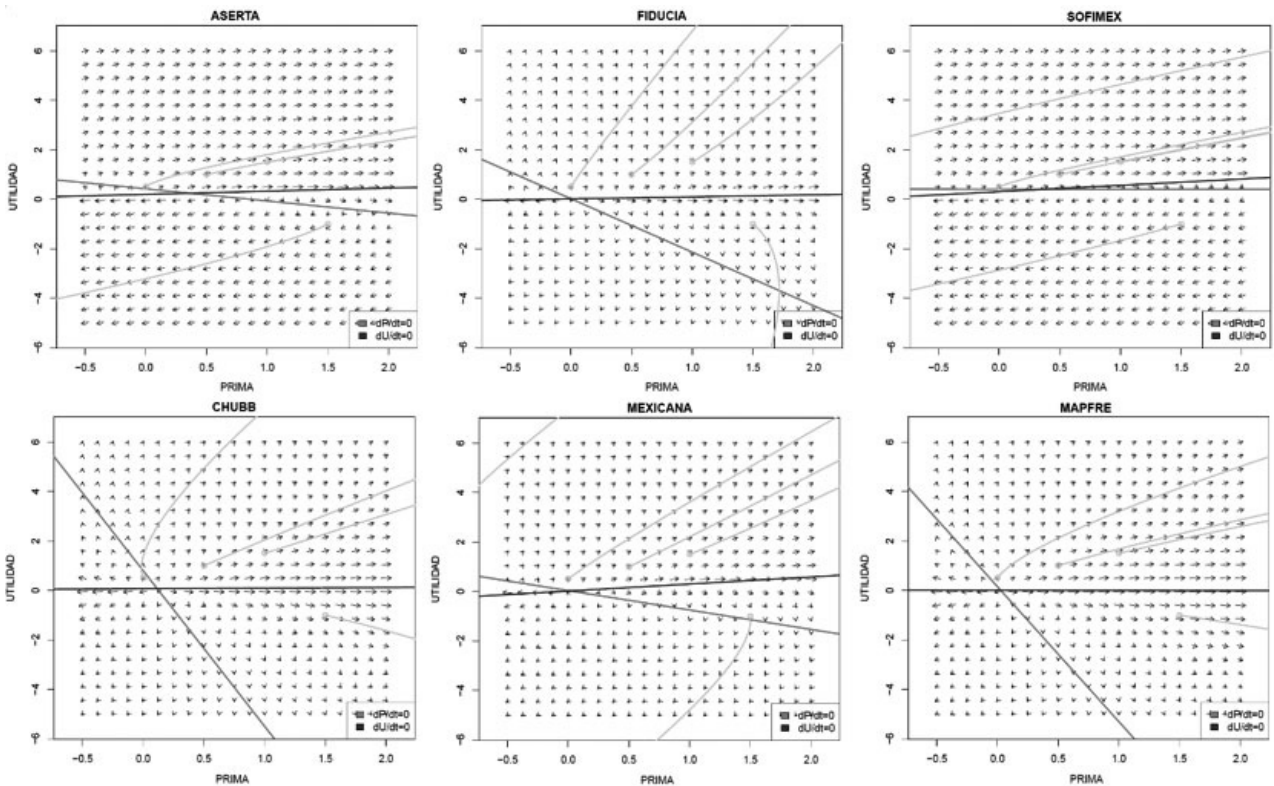

Figura 5. Diagrama de fase para caso 3: Aserta, Fiducia, Sofimex, Chubb, Mexicana y Mapfre.

Fuente: Elaboración propia con base en (Grayling, 2014) R-Project.

Finalmente, las afianzadoras Monterrey, Atlas, Dorama, Inbursa y Primero constituyen el cuarto caso en donde tanto la pendiente de la trayectoria de la utilidad como de las primas son negativas, aunque Inbursa presenta una pendiente cercana a la elasticidad perfecta en su trayectoria de utilidades. En el caso 4 se tiene que cuando se tiene una estrategia agresiva de aumento de primas, las utilidades comienzan a caer. Aunque, todas las afianzadoras de dicho caso tienen la particularidad de que, en la región I, los aumentos de primas ubicados en esa región mueven el sistema de tal forma que cualquier monto de prima o estrategia que se siga, 
provocaría una variación positiva en sus utilidades. En la figura 6 se observa el caso 4, cabe destacar que no se muestra la gráfica de Monterrey ya que esta fue presentada inicialmente para describir la interpretación de los diagramas de fase.
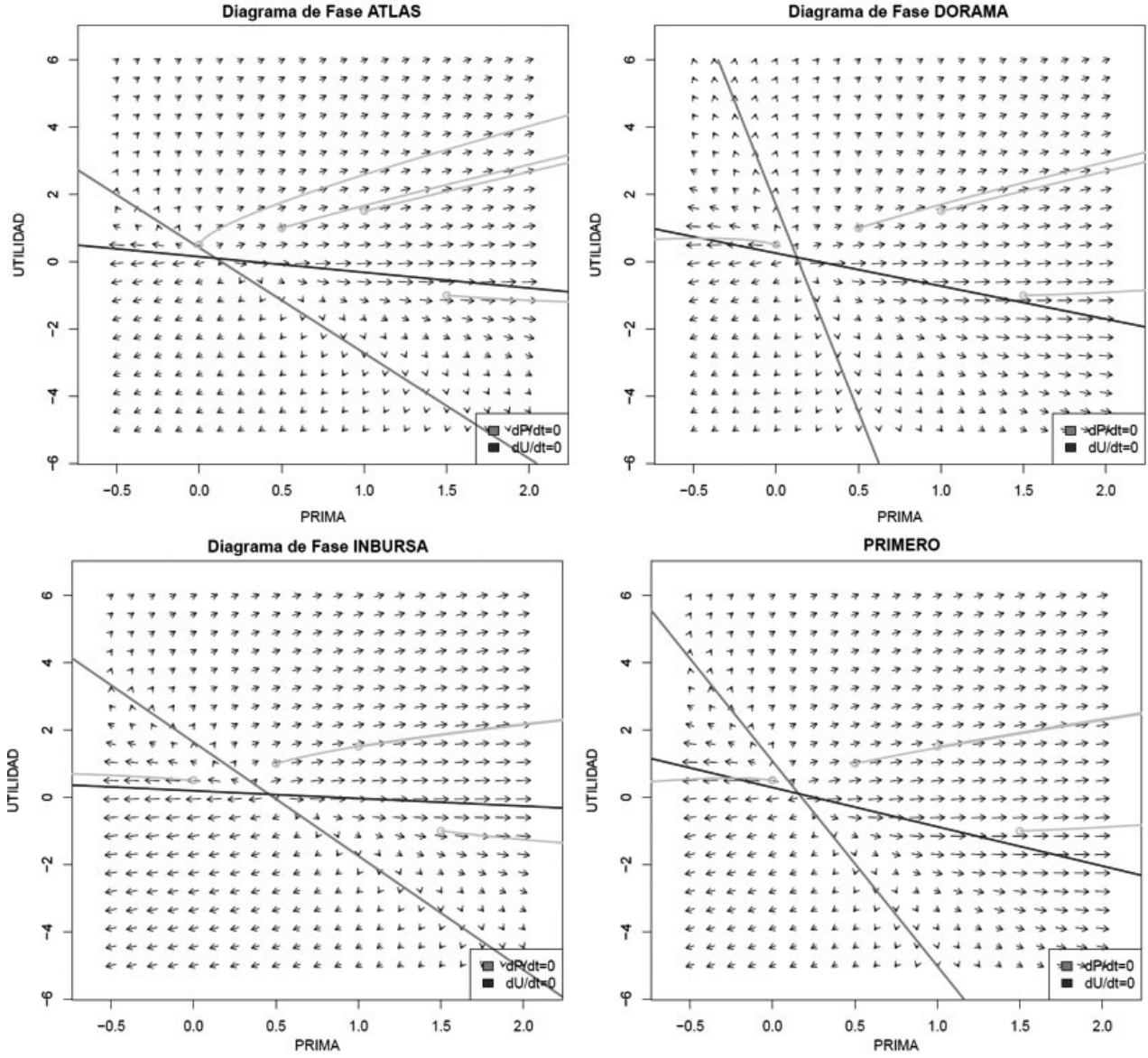

Figura 6.Diagrama de fase para caso 3: Monterrey, Atlas, Dorama, Inbursa y Primero.

Fuente: Elaboración propia con base en (Grayling, 2014) R-Project. 


\section{Conclusiones}

El objetivo del presente trabajo fue analizar la dinámica entre el total de primas emitidas por el sector afianzador y la utilidad bruta reportada por las mismas, en concreto, se propuso el uso de diagramas de fase con la finalidad de comprender la dinámica temporal entre las variables y conocer sus trayectorias en relación al estado estable. En función de las reglas de operación de Solvencia II, se asume que las afianzadoras pequeñas son las que mayor impacto tienen al operar conforme a lo establecido en la nueva regulación, teniendo un impacto directo en el costo de las primas y la generación de utilidades.

A partir de la metodología propuesta se señala que las afianzadoras mexicanas, a pesar de que sus utilidades, presentan comportamientos globalmente inestables (a excepción de Asecam, en donde hay regiones que permiten que la afianzadora regrese a su estado estable), asimismo se verifican los casos donde una mayor aversión al riesgo de las afianzadoras (representadas por las primas emitidas) no siempre reflejan mayores utilidades a menos de que lo hagan de forma agresiva, y eso es para el caso de Monterrey, Atlas, Dorama, Inbursa y Primero. Asimismo, se observaron comportamientos inelásticos como Inbursa, Chubb, Fiducia y Mapfre, presentan una trayectoria en sus utilidades con pendiente cercana de ser perfectamente elástica, por lo que son más sensibles ante variaciones de sus primas.

Vale la pena mencionar que nuestro trabajo se encuentra acotado a la operación de la operación de fianzas en México, que tienen diferencias sustanciales a la forma de operar de las aseguradoras. La relevancia del trabajo es impulsar a las afianzadoras a perseguir un mejor modelo de negocio que les permita incrementar sus utilidades por el buen resultado financiero que puedan obtener y se pretende evidenciar la viabilidad de posibles fusiones o adquisiciones entre ellas, migrando a aseguradoras de caución, por ser claro que la legislación actual no les permite trabajar con las primas para tener márgenes operativos competitivos. La principal aportación del presente trabajo, es la utilización de control óptimo (diagramas de fase) para el análisis de situaciones financieras como es el caso de las afianzadoras; mismo que de forma natural se puede extender al mercado asegurador y al sector financiero en general. 


\section{Referencias}

Álvarez, S. J., \& Londoño, P. J. (2014). Control de velocidad angular constante basado en un sistema splitter pi en una desarrolladora de papel. Revista Politécnica , 10 (18), 115-123.

Belmonte, L. M., Morales, R., Fernández-Caballero, A., \& Somolinos, J. (2016). Robust Decentralized Nonlinear Control for a Twin Rotor MIMo System. Sensors, 16(8), https://doi.org/10.3390/s16081160

Blanchard, O. (1981). Output, the Stock Market, and Interest Rates. The American Economic Review, 71(1), 132-143. Braun, A., Schmeiser, H., \& Schreiber, F. (2017). Portfolio Optimization Under Solvency II: Implicit Constraints Imposed by the Market Risk Standard Formula. Journal of Risk and Insurance, 84(1), 177-207. https://doi. org/10.1111/jori.12077.

Cámara de Diputados, d. H. (1935). Ley Sobre el Contrato de Seguro. México D.F.: Diario Oficial de la Federación.

Cámara de Diputados, d. H. (1950). Ley Federal de Instituciones de Fianzas. México D.F.: Diario Oficial de la Federación, 29 de diciembre de 1950.

Cámara de Diputados, d. H. (2013). Ley de Instituciones de Seguros y de Fianzas. México D.F.: Diario Oficial de la Federación.

Chessab, M. M. (2016). Fuzzy PID controller for nano-satellite attitute control. Journal of Science \& Arts, 16(4),407-416. Chow, G. (1983). Econometrics. New York: McGraw Hill.

CNSF. (21 de 02 de 2018). Circular Única de Seguros y Fianzas. Obtenido de Anexo 22.1.2 Criterios de contabilidad aplicables a las instituciones, sociedades mutualistas y sosiedades controladoras: http://www.cnsf.gob.mx/ CUSFELECTRONICA/CUSF/A_22_1_2

Comisión Nacional de Seguros y Fianzas. (2014). Circular Única de Seguros y Fianzas. México D.F.: Diario Oficial de la Federación.

Commission, E. (27 de 04 de 2017). Business, Economy, Euro. Obtenido de Banking and Finance: http://ec.europa. eu/finance/insurance/solvency/index_en.htm

Eling, M., \& Pankoke, D. (2014). Basis Risk, Procyclicality, and Systemic Risk in the Solvency II Equity Risk Module. Journal of Insurance Regulation, 33 (1), 1-39.

Fianzas, C. N. (2015). Boletín de Análisis Sectorial Seguros y Fianzas. Año 15 No. 55. México D.F.: Comisión Nacional de Seguros y Fianzas.

Gavira, D. N., \& Castillo, R. C. (2017). Solvencia II y el cambio de normatividad en México, retos y perspectivas del sector afianzador. Revista de Investigación en ciencias contables y Administrativas, 43-69.

Grayling, M. (6 de Julio de 2014). phaseR: Phase Plane Analysis of One and Two Dimensional Autonomous ODE Systems. Versión 1.3. Disponible en: https://CRAN.R-project.org/package=phaseR

Hongbin, W., Jian, D., \& Yuelieng, W. (2016). Discrete PID-Type Iterative Learning Control for Mobile Robot. Journal of Control Science and Engineering, 2016. http://dx.doi.org/10.1155/2016/2320746

Laitner, J. (1990). Tax Changes and Phase Diagrams for an Overlapping Generations Model. Journal of Political Economy, 98 (1), 193-220. https://doi.org/10.1086/261675

Lucas, R., \& Sargent, T. (1981). Rational Expectations and Econometric Practice. Minneapolis: University of Minnesota.

Martínez,E., \& Turrubiartes, R. E. (2017). Perspectivas 2017: Sector Asegurador Mexicano. FitchRatings. Disponible en: http://www.fitchratings.mx/ArchivosHTML/RepEsp_13634.pdf.

Muth, J. (1961). Rational Expectations and the Theory of Price Movements. Econometrica, 29(3), 315-335.

PDC, P. D. (1973). Diario Oficial de las Comunidades Europeas 06. Disponible en: https://eur-lex.europa.eu/LexUriServ/LexUriServ.do?uri=CELEX:31973L0239:ES:HTML

PDV. (1979). Gazzetta Ufficiale delle Comunità Europee. Disponible en: https://eur-lex.europa.eu/legal-content/IT/ TXT/PDF/?uri=CELEX:01979L0267-19950807\&from $=\mathrm{GA}$

Saini, P., Kumar, R., \& Rajput, N. (2016). Cascade-PID control of a nonlinear chemical process. Nonlinear Studies, 23(4), 563-570. 
Sargent, T., \& Wallace, N. (1973). Rational Expectations and The Dynamics of Hyperinflation. International Economic Review, 14, 328-350.

Shone, R. (2002). Economic Dynamics: Phase Diagrams and Their Economic Application (2da Ed.). New York: Cambridge University Press.

Sirmans, E. T., \& Mccullough, K. A. (2017). A Comparison of the Risk Management and Own Risk and Solvency Assessment Model Act and Insurer Ratings. Journal of Insurance Regulation , 36(3), 1-23.

Zimmer, A., Grundl, H., Schade, C., \& Glenzer, F. (2016). An incentive-compatible experiment on probabilistic insurance and implications for an insurer's solvency level. Journal of Risk and Insurance, 85(1), 245-273. https://doi.org/10.1111/jori.12148.

Zoujun, W. (2016). Fuzzy Self-Tuning PID Control Method Based on Multi-Agent Particle Swarm Algorithm . International Journal Of Simulation--Systems, Science \& Technology, 17(42),1-6. 